\title{
A MONOMIAL CHAOS APPROACH FOR EFFICIENT UNCERTAINTY QUANTIFICATION IN NONLINEAR PROBLEMS*
}

\author{
JEROEN A. S. WITTEVEEN ${ }^{\dagger}$ AND HESTER BIJL ${ }^{\dagger}$
}

\begin{abstract}
A monomial chaos approach is presented for efficient uncertainty quantification in nonlinear computational problems. Propagating uncertainty through nonlinear equations can be computationally intensive for existing uncertainty quantification methods. It usually results in a set of nonlinear equations which can be coupled. The proposed monomial chaos approach employs a polynomial chaos expansion with monomials as basis functions. The expansion coefficients are solved for using differentiation of the governing equations, instead of a Galerkin projection. This results in a decoupled set of linear equations even for problems involving polynomial nonlinearities. This reduces the computational work per additional polynomial chaos order to the equivalence of a single Newton iteration. Error estimates are derived, and monomial chaos is applied to uncertainty quantification of the Burgers equation and a two-dimensional boundary layer flow problem. The results are compared with results of the Monte Carlo method, the perturbation method, the Galerkin polynomial chaos method, and a nonintrusive polynomial chaos method.
\end{abstract}

Key words. uncertainty quantification, polynomial chaos, computational fluid dynamics, non deterministic approaches

AMS subject classifications. 65C20, 65C 30,65 N30

DOI. $10.1137 / 06067287 \mathrm{X}$

1. Introduction. In practical computational problems, physical parameters and boundary conditions are often subject to uncertainty. Until recently, these physical uncertainties were usually neglected, which resulted in a single deterministic run for the mean values of the uncertain parameters. Nowadays, such a deterministic simulation is no longer adequate for reliable computational predictions. Therefore, there has recently been a growing interest in accounting for physical uncertainties in computational problems [4].

Modeling uncertainty has been common in computational structure mechanics for some time now [15]. Uncertainty analysis in computational fluid dynamics is relatively new $[12,19,24]$. An important characteristic of problems in fluid dynamics is that they are governed by a system of nonlinear partial differential equations. The discretization of these equations for realistic flows results in computational problems involving millions of unknowns. This makes deterministic computational fluid dynamics already computationally intensive. Therefore, it is important to apply uncertainty quantification methods which can deal with the nonlinearities in an efficient way. By developing efficient methods, uncertainty quantification can become economically feasible in practical flow problems.

Physical problems are usually described mathematically in terms of differential equations. The uncertainty in these problems can often be modeled by parametric uncertainties. Therefore, parametric uncertainty is considered in a physical model

${ }^{*}$ Received by the editors October 20, 2006; accepted for publication (in revised form) November 29, 2007; published electronically March 21, 2008. This research is supported by the Technology Foundation STW, applied science division of NWO, and the technology programme of the Ministry of Economic Affairs.

http://www.siam.org/journals/sisc/30-3/67287.html

${ }^{\dagger}$ Department of Aerospace Engineering, Delft University of Technology, Kluyverweg 1, 2629 HS Delft, The Netherlands (j.a.s.witteveen@tudelft.nl, h.bijl@tudelft.nl). 
described by the following differential equation for $u(\mathbf{x}, t, \omega)$ with operator $\mathcal{L}$ and source term $S$ :

$$
\mathcal{L}(\mathbf{x}, t, \alpha(\omega) ; u(\mathbf{x}, t, \omega))=S(\mathbf{x}, t, \alpha(\omega)), \quad \mathbf{x} \in D, t \in[0, T]
$$

with appropriate initial and boundary conditions and $\alpha(\omega)$ an uncertain parameter with a known uncertainty distribution. The argument $\omega$ is used to emphasize the fact that an uncertain variable is a function of the random event $\omega \in \Omega$ of the probability space $(\Omega, \sigma, P)$. Equation (1.1) is an uncertainty quantification problem for the uncertain variable $u(\mathbf{x}, t, \omega)$. Below, four widely used uncertainty quantification methods are briefly reviewed for comparison with the proposed monomial chaos approach: the Monte Carlo method, the perturbation method [10], the Galerkin polynomial chaos method [3], and a nonintrusive polynomial chaos method [9]. For simplicity, the methods are reviewed for the case of a single uncertain parameter $\alpha(\omega)$. They all have extensions to higher dimensions.

The Monte Carlo method. A robust approach to solving (1.1) is the Monte Carlo method. It is based on solving the deterministic problem multiple times for a set of $N$ realizations of the uncertain parameter $\left\{\alpha_{k}\right\}_{k=1}^{N}$ with $\alpha_{k} \equiv \alpha\left(\omega_{k}\right)$,

$$
\mathcal{L}\left(\mathbf{x}, t, \alpha_{k} ; u_{k}(\mathbf{x}, t)\right)=S\left(\mathbf{x}, t, \alpha_{k}\right), \quad k=1, \ldots, N,
$$

with $u_{k}(\mathbf{x}, t) \equiv u\left(\mathbf{x}, t, \omega_{k}\right)$. The stochastic properties of the output can be obtained from the set of $N$ realizations of the uncertain variable $\left\{u_{k}(\mathbf{x}, t)\right\}_{k=1}^{N}$. Due to the slow convergence rate, the standard Monte Carlo approach can be impractical when solving a single deterministic problem already involving a large amount of computational work. Methods exist to improve the convergence rate of standard Monte Carlo, such as Latin-hypercube sampling and variance reduction techniques; see, for example, [6].

The perturbation method. A fast method for determining low-order statistics is the perturbation method (also called the moment method) [7, 10, 16]. It has recently been applied to problems in computational fluid dynamics $[12,17]$. In the perturbation method the statistical moments of the output are expanded around the expected value of the uncertain parameter using Taylor series expansions. These expansions are usually truncated at second order, since for higher orders the equations become extremely complicated $[3,10]$. The second-order estimate of the mean value is given by $[10]$ as

$$
\left.\mathbb{E}[u(\mathbf{x}, t, \omega)] \approx u(\mathbf{x}, t, \omega)\right|_{\alpha=\mu_{\alpha}}+\left.\frac{1}{2} \operatorname{Var}(\alpha(\omega)) \frac{\partial^{2} u}{\partial \alpha^{2}}\right|_{\alpha=\mu_{\alpha}},
$$

with $\mu_{\alpha} \equiv \mathbb{E}[\alpha(\omega)]$. For the first-order approximation, this relation reduces to $\left.\mathbb{E}[u(\mathbf{x}, t, \omega)] \approx u(\mathbf{x}, t, \omega)\right|_{\mu_{\alpha}}$. The first-order estimate of the variance is given as

$$
\operatorname{Var}[u(\mathbf{x}, t, \omega)] \approx\left(\left.\frac{\partial u}{\partial \alpha}\right|_{\alpha=\mu_{\alpha}}\right)^{2} \operatorname{Var}[\alpha(\omega)] .
$$

The moment approximations require the computation of the first and second sensitivity derivatives of the solution $u(\mathbf{x}, t, \omega)$ with respect to the uncertain parameter $\alpha(\omega)$ for $\alpha(\omega)=\mu_{\alpha}$. A method for evaluating these sensitivity derivatives is the continuous sensitivity equation method $[7,16]$. In the continuous sensitivity equation method a differential equation for the $i$ th sensitivity derivative $\left.\frac{\partial^{i} u}{\partial \alpha^{i}}\right|_{\mu_{\alpha}}$ is obtained by implicit 
differentiation of the governing equation (1.1) with respect to $\alpha$ for $\alpha(\omega)=\mu_{\alpha}$. The resulting equation is called the $i$ th continuous sensitivity equation

$$
\left.\frac{\partial^{i}}{\partial \alpha^{i}} \mathcal{L}(\mathbf{x}, t, \alpha(\omega) ; u(\mathbf{x}, t, \omega))\right|_{\mu_{\alpha}}=\left.\frac{\partial^{i}}{\partial \alpha^{i}} S(\mathbf{x}, t, \alpha(\omega))\right|_{\mu_{\alpha}} .
$$

The application of the perturbation method is limited to low-order approximations for small perturbations, i.e., inputs with a small variance. Furthermore, the method cannot readily be extended to compute the probability distribution function of the response process $[3,10]$.

The Galerkin polynomial chaos method. A method that is not limited to loworder statistics and small perturbations is the polynomial chaos expansion introduced by Ghanem and Spanos [3]. The method has recently been applied to computational fluid dynamics $[19,24]$. The polynomial chaos expansion is a polynomial expansion of orthogonal polynomials in terms of random variables to approximate the uncertainty distribution of the output. The method is based on the homogeneous chaos theory of Wiener [21]. The homogeneous polynomial chaos expansion, which is based on Hermite polynomials and Gaussian random variables, can approximate any functional in $L_{2}(C)$ and converges in the $L_{2}(C)$ sense [2]. It can achieve an exponential convergence rate for Gaussian input distributions due to the orthogonality of the Hermite polynomials with respect to the Gaussian measure. The exponential convergence has been extended to other input distributions by employing other basis polynomials $[20,22,23]$. The expansion coefficients are determined in the context of a stochastic finite element approach by using a Galerkin projection in probability space. The polynomial chaos expansions of the uncertain input parameter $\alpha(\omega)$ and the uncertain solution $u(\mathbf{x}, t, \omega)$ are

$$
\alpha(\omega)=\sum_{j=0}^{1} \alpha_{j} \Phi_{j}(\xi(\omega)), \quad u(\mathbf{x}, t, \omega)=\sum_{i=0}^{\infty} u_{i}(\mathbf{x}, t) \Phi_{i}(\xi(\omega)),
$$

where $\left\{\Phi_{i}(\xi)\right\}_{i=0}^{\infty}$ is a set of orthogonal polynomials and the random variable $\xi(\omega)$ is given by a linear transformation of $\alpha(\omega)$ to an appropriate standard domain, i.e. $[-1,1],[0, \infty)$, or $(-\infty, \infty)$. Due to this linear transformation the polynomial chaos expansion of $\alpha(\omega)$ in (1.6) is exact within the first two terms. For the numerical implementation the polynomial chaos expansion for $u(\mathbf{x}, t, \omega)$ in $(1.6)$ is truncated to $(p+1)$ terms, where $p$ is the polynomial chaos order of the approximation. Substituting the truncated expansions into (1.1) and performing a Galerkin projection onto each polynomial basis $\left\{\Phi_{i}(\xi)\right\}_{i=0}^{p}$ results in a coupled set of $(p+1)$ deterministic equations

$$
\left\langle\mathcal{L}\left(\mathbf{x}, t, \sum_{j=0}^{1} \alpha_{j} \Phi_{j} ; \sum_{i=0}^{p} u_{i} \Phi_{i}\right), \Phi_{k}\right\rangle=\left\langle S\left(\mathbf{x}, t, \sum_{j=0}^{1} \alpha_{j} \Phi_{j}\right), \Phi_{k}\right\rangle
$$

for $k=0,1, \ldots, p$. This system of equations can be solved using standard iterative methods [5]. The Galerkin polynomial chaos method can be intrusive to implement and computationally intensive to solve, due to the coupled set of equations (1.7).

A nonintrusive polynomial chaos method. To avoid solving a coupled set of equations, a nonintrusive polynomial chaos method can be used. It approximates the polynomial chaos coefficients by solving a series of deterministic problems. An example of a nonintrusive polynomial chaos method is the method of Hosder and 
Walters (see $[9,18])$. The polynomial chaos expansion coefficients $\left\{u_{k}(\mathbf{x}, t)\right\}_{k=0}^{p}$ in (1.6) are approximated by evaluating the deterministic problem at $(p+1)$ points in random space $\left\{\xi_{k}\right\}_{k=0}^{p}$, with $\xi_{k} \equiv \xi\left(\omega_{k}\right)$,

$$
\mathcal{L}\left(\mathbf{x}, t, \alpha_{k} ; u_{k}^{*}(\mathbf{x}, t)\right)=S\left(\mathbf{x}, t, \alpha_{k}\right), \quad k=0,1, \ldots, p,
$$

where $u_{k}^{*}(\mathbf{x}, t)$ is the realization of $u(\mathbf{x}, t, \omega)$ for $\alpha(\omega)=\alpha_{k}$. The polynomial chaos coefficients $\left\{u_{k}(\mathbf{x}, t)\right\}_{k=0}^{p}$ are then approximated by the following relatively small linear system:

$$
\left(\begin{array}{cccc}
\Phi_{0}\left(\xi_{0}\right) & \Phi_{1}\left(\xi_{0}\right) & \cdots & \Phi_{p}\left(\xi_{0}\right) \\
\Phi_{0}\left(\xi_{1}\right) & \Phi_{1}\left(\xi_{1}\right) & \cdots & \Phi_{p}\left(\xi_{1}\right) \\
\vdots & \vdots & \ddots & \vdots \\
\Phi_{0}\left(\xi_{p}\right) & \Phi_{1}\left(\xi_{p}\right) & \cdots & \Phi_{p}\left(\xi_{p}\right)
\end{array}\right)\left(\begin{array}{c}
u_{0}(\mathbf{x}, t) \\
u_{1}(\mathbf{x}, t) \\
\vdots \\
u_{p}(\mathbf{x}, t)
\end{array}\right)=\left(\begin{array}{c}
u_{0}^{*}(\mathbf{x}, t) \\
u_{1}^{*}(\mathbf{x}, t) \\
\vdots \\
u_{p}^{*}(\mathbf{x}, t)
\end{array}\right)
$$

which can be solved using a single LU decomposition. This nonintrusive polynomial chaos method can be shown to converge to the Galerkin polynomial chaos expansion coefficients under certain conditions [9]. As for the Monte Carlo method (1.2), nonintrusive polynomial chaos results in a set of equations (1.8) which coincide with the deterministic problem for varying parameter values. However, the number of deterministic evaluations can be orders of magnitude smaller than for a standard Monte Carlo simulation due to the combination with the polynomial chaos expansion.

Compared to solving the problem deterministically, using a nonintrusive polynomial chaos method results in a multiplication of computational work by a factor $(p+1)$. For computationally very intensive problems this increase of computational work can be a major drawback for the application of uncertainty quantification. Consider, for example, practical applications of nonlinear computational fluid dynamics in time dependent problems involving complex geometries. These deterministic problems can already take weeks or even longer to solve. An increase of this amount of computational work by a factor $(p+1)$ is significant. Especially in iterative design processes of industrial applications this can make uncertainty quantification impractical. On the other hand, uncertainty quantification is in these cases essential for robust design optimization. Therefore, there is a need for a further reduction of the computational costs of uncertainty quantification methods.

In this paper, a monomial chaos approach is proposed to reduce the costs of uncertainty quantification in computationally intensive nonlinear problems. The method employs the polynomial chaos expansion with monomials as basis functions. The monomial chaos expansion coefficients are solved for using differentiation of the governing equations, instead of a Galerkin projection. This results in a decoupled set of linear equations even for problems involving polynomial nonlinearities. This reduces the computational work per additional polynomial chaos order to the equivalence of a single Newton iteration. Therefore, monomial chaos can be a computationally efficient alternative for existing uncertainty quantification methods in nonlinear problems. The monomial chaos approach is introduced in this paper for one uncertain input parameter to demonstrate the properties of the method and to make a basic comparison with other uncertainty quantification methods. The extension of monomial chaos to multiple uncertain parameters and random fields is briefly addressed.

The paper is organized as follows. The monomial chaos is introduced and error estimates derived in section 2. In section 3 the monomial chaos is applied to 
the Burgers equation to demonstrate the properties of the proposed approach for a standard nonlinear advection-diffusion test problem in one dimension. The results are compared with results of the perturbation method, the Galerkin polynomial chaos method, and the nonintrusive polynomial chaos method in section 4 . In section 5 the monomial chaos is applied to a two-dimensional boundary layer flow problem as an example of a standard nonlinear test problem from two-dimensional incompressible fluid dynamics. In section 6 the conclusions are summarized.

2. The monomial chaos approach. In this section the monomial chaos approach is proposed. In section 2.1 the monomial chaos approach is introduced in general as applied to (1.1). Error estimates are given in section 2.2.

2.1. The monomial chaos formulation. The monomial chaos approach employs a polynomial chaos expansion with monomials as basis functions to determine the uncertainty distribution of the output. The equations for the monomial chaos expansion coefficients are obtained by differentiating the deterministic equation with respect to the uncertain input parameter. This results in a decoupled set of $(p+1)$ equations for the $(p+1)$ coefficients of a $p$ th-order monomial chaos expansion, in which each equation solves for a monomial chaos coefficient sequentially. Due to the product rule the differentiation of the governing equations also results in a set of linear equations even for problems involving polynomial nonlinearities. This reduces the additional computational work per polynomial chaos order to the equivalence of a single Newton iteration. Therefore, monomial chaos can be an efficient alternative for uncertainty quantification in computationally intensive nonlinear problems.

Consider the application of monomial chaos to a physical model involving polynomial nonlinearities and parametric uncertainty given by (1.1),

$$
\mathcal{L}(\mathbf{x}, t, \alpha(\omega) ; u(\mathbf{x}, t, \omega))=S(\mathbf{x}, t, \alpha(\omega))
$$

The uncertain parameter $\alpha(\omega)$ and the solution $u(\mathbf{x}, t, \omega)$ are expanded into a polynomial chaos expansion

$$
\alpha(\omega)=\sum_{j=0}^{1} \alpha_{j} \Psi_{j}(\xi(\omega)), \quad u(\mathbf{x}, t, \omega)=\sum_{i=0}^{\infty} u_{i}(\mathbf{x}, t) \Psi_{i}(\xi(\omega)),
$$

where the random variable $\xi(\omega)$ is given by a linear transformation of the uncertain input parameter $\alpha(\omega)$ to an appropriate standard domain, i.e., $[-1,1],[0, \infty)$, or $(-\infty, \infty)[23]$. Due to this linear transformation the polynomial chaos expansion of $\alpha(\omega)$ in $(2.1)$ is exact within the first two terms. In the monomial chaos the basis polynomials $\left\{\Psi_{i}(\xi)\right\}_{i=0}^{\infty}$ are monomials around $\xi(\omega)=\mu_{\xi}$ with $\mu_{\xi} \equiv \mathbb{E}[\xi(\omega)]$ :

$$
\Psi_{i}(\xi(\omega))=\left(\xi(\omega)-\mu_{\xi}\right)^{i}, \quad i=0,1, \ldots
$$

These monomials are chosen as basis functions because they satisfy the property

$$
\left.\frac{\mathrm{d}^{j} \Psi_{i}}{\mathrm{~d} \xi^{j}}\right|_{\xi=\mu_{\xi}}=\left\{\begin{array}{cc}
i !, & i=j, \\
0, & i \neq j,
\end{array}\right.
$$

which says that taking the $j$ th derivative of the monomials $\left\{\Psi_{i}(\xi)\right\}_{i=0}^{\infty}$ with respect to $\xi$ at $\xi(\omega)=\mu_{\xi}$ results in a nonzero term for $i=j$ only. This property of monomials results in the decoupled set of equations for the monomial chaos coefficients $\left\{u_{i}(\mathbf{x}, t)\right\}$. 
Substitution of the monomial chaos expansions (2.1) with (2.2) into the governing equation (1.1) results in

$$
\mathcal{L}\left(\mathbf{x}, t, \sum_{j=0}^{1} \alpha_{j} \Psi_{j}(\xi) ; \sum_{i=0}^{\infty} u_{i}(\mathbf{x}, t) \Psi_{i}(\xi)\right)=S\left(\mathbf{x}, t, \sum_{j=0}^{1} \alpha_{j} \Psi_{j}(\xi)\right) .
$$

To obtain a set of equations for the expansion coefficients of the solution $\left\{u_{i}(\mathbf{x}, t)\right\}$, (2.4) is differentiated with respect to $\xi$ for $\xi(\omega)=\mu_{\xi}$. Taking the $k$ th derivative of (2.4) results in an equation for the $k$ th expansion coefficient $u_{k}(\mathbf{x}, t)$,

$$
\begin{array}{r}
\left.\frac{\partial^{k}}{\partial \xi^{k}} \mathcal{L}\left(\mathbf{x}, t, \sum_{j=0}^{1} \alpha_{j} \Psi_{j}(\xi) ; \sum_{i=0}^{\infty} u_{i}(\mathbf{x}, t) \Psi_{i}(\xi)\right)\right|_{\xi=\mu_{\xi}} \\
=\left.\frac{\partial^{k}}{\partial \xi^{k}} S\left(\mathbf{x}, t, \sum_{j=0}^{1} \alpha_{j} \Psi_{j}(\xi)\right)\right|_{\xi=\mu_{\xi}}
\end{array}
$$

for $k=0,1, \ldots$. This set of equations can be discretized using standard discretization techniques [8]. Due to the combination of differentiation of (2.4) and property (2.3), all higher-order coefficients $\left\{u_{i}(\mathbf{x}, t)\right\}_{i=k+1}^{\infty}$ drop out of the equation, which results in a decoupled set of equations for $u_{k}(\mathbf{x}, t)$ in terms of $\left\{u_{i}(\mathbf{x}, t)\right\}_{i=0}^{k-1}$. This is illustrated in section 3 where the monomial chaos is applied to the Burgers equation. Furthermore, the decoupled set of equations (2.5) is linear in $u_{k}(\mathbf{x}, t)$ due to the product rule in differentiation, even if the governing equation (1.1) contains polynomial nonlinearities (except for $k=0$ ). The equation for $k=0$ coincides with the deterministic problem for the expected value of the uncertain parameter $\mu_{\alpha}$. For nonlinear problems solved using Newton linearization, the additional computational work per polynomial chaos order is proportional to one Newton iteration.

A $p$ th-order monomial chaos approximation of the solution $u(\mathbf{x}, t, \omega)$ is given by truncating the monomial chaos expansion for $u(\mathbf{x}, t, \omega)$ in $(2.1)$ at $p$. The monomial chaos coefficients $\left\{\alpha_{j}\right\}_{j=0}^{1}$ of the uncertain parameter $\alpha(\omega)$ with a known uncertainty distribution can be determined by differentiating the monomial chaos expansion for $\alpha(\omega)$ in (2.1) with respect to $\xi$ for $\xi(\omega)=\mu_{\xi}$, which results, using property (2.3), in

$$
\alpha_{j}=\left.\frac{1}{j !} \frac{\mathrm{d}^{j} \alpha}{\mathrm{d} \xi^{j}}\right|_{\xi=\mu_{\xi}}, \quad j=0,1
$$

where $\left.\frac{\mathrm{d}^{j} \alpha}{\mathrm{d} \xi^{j}}\right|_{\mu_{\xi}}$ is known and $\alpha_{0}=\mu_{\alpha}$.

Equations (2.5) are similar to the continuous sensitivity equations (1.5) of the perturbation method, which are obtained by implicit differentiation. The monomial chaos method can be viewed as an extension of the perturbation method, which is usually limited to second-order approximations of the first two moments. The monomial chaos approach can be employed for obtaining higher-order approximations of the uncertainty distribution and the statistical moments of the output at computational costs equivalent to those of the perturbation method.

On the other hand, in the monomial chaos approach the uncertain parameter and variables are expanded in a polynomial expansion as in the polynomial chaos method, using monomials instead of orthogonal polynomials in the polynomial chaos method. 
The monomial chaos approach can therefore be applied to the same set of arbitrary input probability distributions as the polynomial chaos method. The outputs of the monomial chaos approach are higher-order approximations of the distribution and the statistical moments by solving a decoupled set of linear equations, instead of a possibly coupled set of nonlinear equations in the polynomial chaos method.

Next to the relatively low computational work per polynomial chaos order the monomial chaos has additional advantages which are important for practical applications. First, the polynomial chaos order of the monomial chaos approximation can be determined during the computation while solving for the higher-order coefficients sequentially. Second, the equations (2.5) depend only on the mean value of the uncertain input parameter $\mu_{\alpha}$. Therefore, the influence of different input uncertainty distributions and variances can be studied after solving (2.5) once. This is an important property since in practical problems the input distribution itself can also be subject to uncertainty.

The monomial chaos is moderately intrusive, since for solving (2.5) the summation of the matrix and vector entries in the deterministic solver have to be modified. For decreasing the intrusiveness of the monomial chaos, the differentiation of the governing equations can be replaced by finite difference differentiation in random space.

Here, the monomial chaos approach is considered for a single uncertain input parameter. The monomial chaos approach can be extended to multiple uncertain input parameters by introducing a vector of random variables $\boldsymbol{\xi}(\omega)=\left(\xi_{1}(\omega), \ldots, \xi_{n}(\omega)\right)$, where $n$ is the number of uncertain input parameters. The basis consists in that case of multidimensional monomials $\boldsymbol{\Psi}_{i}(\boldsymbol{\xi}(\omega)$ ), which are tensor products of the onedimensional monomials $\Psi_{i}\left(\xi_{j}(\omega)\right)$. The set of equations for the monomial chaos coefficients (2.5) is then derived using mixed partial derivatives of (2.4) with respect to the random variables $\left\{\xi_{j}(\omega)\right\}_{j=0}^{n}$.

The uncertainty quantification methods reviewed in section 1 can also be extended to multiple uncertain input parameters. For comparison, in the extension of the perturbation method to multiple uncertain input parameters, the statistical moments of the output are expanded around the expected value of the uncertain parameters using multidimensional Taylor series expansions. The polynomial chaos method can be extended to $n$ uncertain parameters by using a multidimensional polynomial chaos expansion in (1.6). The multidimensional polynomial chaos expansion is based on a vector of random variables $\boldsymbol{\xi}(\omega)=\left(\xi_{1}(\omega), \ldots, \xi_{n}(\omega)\right)$ and multidimensional orthogonal polynomials $\boldsymbol{\Phi}_{i}(\boldsymbol{\xi}(\omega))$.

A random field can be handled by the monomial chaos method by first representing the random field in terms of a finite number of independent random input parameters using a Karhunen-Loève expansion [11] as in the polynomial chaos method. For a random field with a relatively high spatial correlation, the number of random input parameters needed to reach a reasonable accuracy with the Karhunen-Loève expansion can be small. In that case the monomial chaos method can be applied to the random input parameters to resolve the effect of the random field. For random fields and random processes with low correlation the required number of random input parameters can be much higher, and approaches other than the monomial chaos method or the polynomial chaos method can be more competitive.

2.2. Error estimates. In this section error estimates for the monomial chaos approach are derived. For simplicity the arguments $\mathbf{x}$ and $t$ are dropped. After computing the monomial chaos coefficients in (2.5), approximations of the mean $\mu_{\mathrm{u}}$, variance $\sigma_{\mathrm{u}}^{2}$, higher-order moments, and the distribution function can be derived. If 
the uncertain variable $u(\omega)$ is expanded in an infinite monomial chaos series, the mean $\mu_{\mathrm{u}}$ is given in terms of the monomial chaos coefficients $\left\{u_{i}\right\}_{i=0}^{\infty}$ by

$$
\mu_{\mathrm{u}}=\sum_{i=0}^{\infty} u_{i} \mu_{\xi, i}
$$

with $\mu_{\xi, i}$ the $i$ th central statistical moment of $\xi(\omega)$,

$$
\mu_{\xi, i}=\int_{\operatorname{supp}(\xi)} \Psi_{i}(\xi) p_{\xi}(\xi) \mathrm{d} \xi
$$

with $\mu_{\xi, 0}=1, \mu_{\xi, 1}=0$ and where $\operatorname{supp}(\xi)$ and $p_{\xi}(\xi)$ are the support and the probability density of $\xi(\omega)$, respectively. The variance $\sigma_{\mathrm{u}}^{2}$ is given by

$$
\sigma_{\mathrm{u}}^{2}=\sum_{i=0}^{\infty} \sum_{j=0}^{\infty} \tilde{u}_{i} \tilde{u}_{j} \mu_{\xi, i+j},
$$

with

$$
\tilde{u}_{i}= \begin{cases}u_{i}-\mu_{\mathrm{u}}, & i=0, \\ u_{i}, & i=1,2, \ldots\end{cases}
$$

In the numerical implementation the infinite series in (2.7) and (2.9) are truncated at a polynomial chaos order $p$. The errors in the approximation in the mean $\varepsilon_{\mu_{u}}$ and the variance $\varepsilon_{\sigma_{\mathrm{u}}^{2}}$ due to the truncation of the monomial chaos expansion are then given by

$$
\varepsilon_{\mu_{\mathrm{u}}}=-\sum_{i=p+1}^{\infty} u_{i} \mu_{\xi, i}
$$

and

$$
\varepsilon_{\sigma_{\mathrm{u}}^{2}}=-2 \sum_{i=p+1}^{\infty} \sum_{j=p+1}^{\infty} u_{i} u_{j} \mu_{\xi, i+j} .
$$

If the monomial chaos coefficients $u_{i}$ decrease fast enough with $i$ for $i=p+1, p+2, \ldots$, such that the leading truncation error term is due to neglecting the $(p+1)$ th coefficient, then the truncation errors can be estimated as

$$
\varepsilon_{\mu_{\mathrm{u}}} \approx-u_{p+1} \mu_{\xi, p+1}
$$

and

$$
\varepsilon_{\sigma_{\mathrm{u}}^{2}} \approx-2 u_{p+1}^{2} \mu_{\xi, p+1}^{2},
$$

which says that the leading error term in the approximation of the variance $\sigma_{\mathrm{u}}^{2}$ results in an underestimation. These a posteriori error estimates can be used in a stopping criterion for determining the polynomial chaos order $p$ of the monomial chaos approximation.

Another contribution to the error in the mean $\mu_{\mathrm{u}}$ and the variance $\sigma_{\mathrm{u}}^{2}$ can be due to the divergence of the monomial chaos expansion in a part of the domain of $\xi(\omega)$. In 
case of an input distribution with an infinite support, i.e., $\xi(\omega) \in(-\infty, \infty)$, there is always a domain $\xi(\omega) \in\left(-\infty, \xi^{-}\right] \cup\left[\xi^{+}, \infty\right)$ in which the monomial chaos expansion of $u(\omega),(2.1)$, diverges. However, it is demonstrated in the following propositions that the divergence of the monomial chaos expansion in $\xi(\omega) \in\left(-\infty, \xi^{-}\right] \cup\left[\xi^{+}, \infty\right)$ results in errors $\tilde{\varepsilon}_{\mu_{\mathrm{u}}}, \tilde{\varepsilon}_{\sigma_{\mathrm{u}}^{2}}$ which are in general small with respect to the truncation errors $\varepsilon_{\mu_{\mathrm{u}}}$ and $\varepsilon_{\sigma_{\mathrm{u}}^{2}}$, and the mean $\mu_{\mathrm{u}}$ and variance $\sigma_{\mathrm{u}}^{2}$.

Proposition 2.1. Let $\xi(\omega) \in(-\infty, \infty)$ and let $\xi(\omega) \in\left(\xi^{-}, \xi^{+}\right)$be the domain of convergence of the monomial chaos expansion of $u(\omega)$, (2.1). If the probability density $p_{\xi}(\xi)$ of $\xi(\omega)$ decreases fast enough as $\xi \rightarrow \pm \infty$ such that

$$
\sum_{i=0}^{\infty}\left|u_{i}\right|\left|\int_{\left(-\infty, \xi^{-}\right] \cup\left[\xi^{+}, \infty\right)} \Psi_{i}(\xi) p_{\xi}(\xi) \mathrm{d} \xi\right| \ll\left|\sum_{i=p+1}^{\infty} u_{i} \int_{\xi^{-}}^{\xi^{+}} \Psi_{i}(\xi) p_{\xi}(\xi) \mathrm{d} \xi\right|,
$$

then the error in the monomial chaos approximation of the mean $\mu_{\mathrm{u}}$ due to the divergence of the monomial chaos expansion in $\xi(\omega) \in\left(-\infty, \xi^{-}\right] \cup\left[\xi^{+}, \infty\right)$ is small compared to the truncation error; i.e., $\left|\tilde{\varepsilon}_{\mu_{\mathrm{u}}}\right| \ll\left|\varepsilon_{\mu_{\mathrm{u}}}\right|$.

Proof. The error $\tilde{\varepsilon}_{\mu_{\mathrm{u}}}$ in the monomial chaos approximation of the mean $\mu_{\mathrm{u}}$ due to the divergence in $\xi(\omega) \in\left(-\infty, \xi^{-}\right] \cup\left[\xi^{+}, \infty\right)$ is defined as

$$
\tilde{\varepsilon}_{\mu_{\mathrm{u}}}=\sum_{i=0}^{\infty} u_{i} \int_{\left(-\infty, \xi^{-}\right] \cup\left[\xi^{+}, \infty\right)} \Psi_{i}(\xi) p_{\xi}(\xi) \mathrm{d} \xi
$$

with

$$
\left|\sum_{i=0}^{\infty} u_{i} \int_{\left(-\infty, \xi^{-}\right] \cup\left[\xi^{+}, \infty\right)} \Psi_{i}(\xi) p_{\xi}(\xi) \mathrm{d} \xi\right| \leq \sum_{i=0}^{\infty}\left|u_{i}\right|\left|\int_{\left(-\infty, \xi^{-}\right] \cup\left[\xi^{+}, \infty\right)} \Psi_{i}(\xi) p_{\xi}(\xi) \mathrm{d} \xi\right| .
$$

The truncation error $\varepsilon_{\mu_{\mathrm{u}}}$ in the monomial chaos approximation of the mean $\mu_{\mathrm{u}}$ due to the truncation of the monomial chaos expansion at $p$ is given by (2.11),

$$
\begin{aligned}
\varepsilon_{\mu_{\mathrm{u}}} & =-\sum_{i=p+1}^{\infty} u_{i} \int_{-\infty}^{\infty} \Psi_{i}(\xi) p_{\xi}(\xi) \mathrm{d} \xi \\
& =-\sum_{i=p+1}^{\infty} u_{i} \int_{\left(-\infty, \xi^{-}\right] \cup\left[\xi^{+}, \infty\right)} \Psi_{i}(\xi) p_{\xi}(\xi) \mathrm{d} \xi-\sum_{i=p+1}^{\infty} u_{i} \int_{\xi^{-}}^{\xi^{+}} \Psi_{i}(\xi) p_{\xi}(\xi) \mathrm{d} \xi
\end{aligned}
$$

with

$$
\left|\sum_{i=p+1}^{\infty} u_{i} \int_{\left(-\infty, \xi^{-}\right] \cup\left[\xi^{+}, \infty\right)} \Psi_{i}(\xi) p_{\xi}(\xi) \mathrm{d} \xi\right| \leq \sum_{i=0}^{\infty}\left|u_{i}\right|\left|\int_{\left(-\infty, \xi^{-}\right] \cup\left[\xi^{+}, \infty\right)} \Psi_{i}(\xi) p_{\xi}(\xi) \mathrm{d} \xi\right| .
$$

According to the assumption, $\left|\tilde{\varepsilon}_{\mu_{\mathrm{u}}}\right| \ll\left|\varepsilon_{\mu_{\mathrm{u}}}\right|$.

Proposition 2.2. Let $\xi(\omega) \in(-\infty, \infty)$, and let $\xi(\omega) \in\left(\xi^{-}, \xi^{+}\right)$be the domain of convergence of the monomial chaos expansion of $u(\omega)$, (2.1). If (i) the probability density $p_{\xi}(\xi)$ of $\xi(\omega)$ decreases fast enough as $\xi \rightarrow \pm \infty$ such that

$$
\left|\sum_{i=0}^{\infty} u_{i} \int_{\left(-\infty, \xi^{-}\right] \cup\left[\xi^{+}, \infty\right)} \Psi_{i}(\xi) p_{\xi}(\xi) \mathrm{d} \xi\right| \ll\left|\sum_{i=0}^{\infty} u_{i} \int_{\xi^{-}}^{\xi^{+}} \Psi_{i}(\xi) p_{\xi}(\xi) \mathrm{d} \xi\right|,
$$

Copyright $@$ by SIAM. Unauthorized reproduction of this article is prohibited. 
or (ii) the probability density $p_{\xi}(\xi)$ of $\xi(\omega)$ decreases fast enough as $\xi \rightarrow \pm \infty$ such that (2.15) holds and the probability density $p_{\xi}(\xi)$ of $\xi(\omega)$ decreases fast enough as $\xi \rightarrow \pm \infty$ such that

$$
\left|\sum_{i=p+1}^{\infty} u_{i} \int_{-\infty}^{\infty} \Psi_{i}(\xi) p_{\xi}(\xi) \mathrm{d} \xi\right| \ll\left|\sum_{i=0}^{p} u_{i} \int_{-\infty}^{\infty} \Psi_{i}(\xi) p_{\xi}(\xi) \mathrm{d} \xi\right|,
$$

then $\left|\tilde{\varepsilon}_{\mu_{\mathrm{u}}}\right| \ll\left|\mu_{\mathrm{u}}\right|$.

Proof. The error $\tilde{\varepsilon}_{\mu_{\mathrm{u}}}$ in the monomial chaos approximation of the mean $\mu_{\mathrm{u}}$ due to the divergence in $\xi(\omega) \in\left(-\infty, \xi^{-}\right] \cup\left[\xi^{+}, \infty\right)$ is given by $(2.16)$,

$$
\tilde{\varepsilon}_{\mu_{\mathrm{u}}}=\sum_{i=0}^{\infty} u_{i} \int_{\left(-\infty, \xi^{-}\right] \cup\left[\xi^{+}, \infty\right)} \Psi_{i}(\xi) p_{\xi}(\xi) \mathrm{d} \xi
$$

The mean $\mu_{\mathrm{u}}$ is given by (2.7) and (2.8), which can be written as

$$
\mu_{\mathrm{u}}=\sum_{i=0}^{\infty} u_{i} \int_{\left(-\infty, \xi^{-}\right] \cup\left[\xi^{+}, \infty\right)} \Psi_{i}(\xi) p_{\xi}(\xi) \mathrm{d} \xi+\sum_{i=0}^{\infty} u_{i} \int_{\xi^{-}}^{\xi^{+}} \Psi_{i}(\xi) p_{\xi}(\xi) \mathrm{d} \xi .
$$

According to assumption (i), $\left|\tilde{\varepsilon}_{\mu_{\mathrm{u}}}\right| \ll\left|\mu_{\mathrm{u}}\right|$. The mean $\mu_{\mathrm{u}}$ based on an infinite monomial chaos series expansion of $u(\omega)$ is given by (2.7) and (2.8), which can also be written as

$$
\mu_{\mathrm{u}}=\sum_{i=0}^{p} u_{i} \int_{-\infty}^{\infty} \Psi_{i}(\xi) p_{\xi}(\xi) \mathrm{d} \xi+\sum_{i=p+1}^{\infty} u_{i} \int_{-\infty}^{\infty} \Psi_{i}(\xi) p_{\xi}(\xi) \mathrm{d} \xi
$$

The error $\varepsilon_{\mu_{\mathrm{u}}}$ in the monomial chaos approximation of the mean $\mu_{\mathrm{u}}$ due to the truncation of the monomial chaos expansion at $p$ is given by (2.11),

$$
\varepsilon_{\mu_{\mathrm{u}}}=-\sum_{i=p+1}^{\infty} u_{i} \int_{-\infty}^{\infty} \Psi_{i}(\xi) p_{\xi}(\xi) \mathrm{d} \xi
$$

According to assumption (ii), we have $\left|\varepsilon_{\mu_{\mathrm{u}}}\right| \ll\left|\mu_{\mathrm{u}}\right|$. The result of Proposition 2.1 gives $\left|\tilde{\varepsilon}_{\mu_{\mathrm{u}}}\right| \ll\left|\mu_{\mathrm{u}}\right|$.

An example of a probability distribution that can satisfy the assumptions of Propositions 2.1 and 2.2 is the Gaussian distribution with density function $p_{\xi}(\xi)=$ $\left(1 / \sqrt{2 \pi \sigma_{\xi}^{2}}\right) \exp \left(-\left(\xi-\mu_{\xi}\right)^{2} /\left(2 \sigma_{\xi}^{2}\right)\right)$, and $\mu_{\xi}$ and $\sigma_{\xi}^{2}$ the mean and variance of $\xi(\omega)$, respectively. This probability density function is exponentially decreasing as $\xi \rightarrow \pm \infty$. Whether a given Gaussian probability distribution satisfies the assumptions depends on the combination of a not too large variance of the uncertain input parameter through $\sigma_{\xi}^{2}$ and sufficient regularity of the uncertain variable $u(\omega)$ through $\left\{u_{i}\right\}_{i=0}^{\infty}$, $\xi^{-}$, and $\xi^{+}$. One can use (2.15), (2.20), and (2.21) to verify whether the monomial chaos expansion of a certain order $p$ is appropriate to use in a particular application. Similar propositions hold for the variance $\sigma_{\mathrm{u}}^{2}$ and the errors $\tilde{\varepsilon}_{\sigma_{\mathrm{u}}^{2}}$ and $\varepsilon_{\sigma_{\mathrm{u}}^{2}}$.

3. Application of monomial chaos. In this section the monomial chaos is applied to the Burgers equation. The test problem is intended for demonstrating the properties of monomial chaos applied to a nonlinear problem and for comparing the results to those of other methods. The Burgers equation is often used to study 


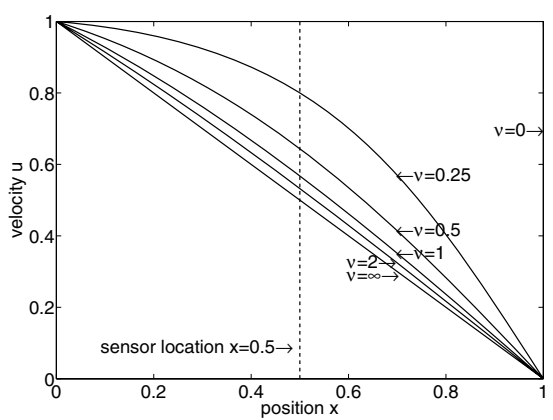

FIG. 1. Deterministic solution of the nonlinear advection-diffusion problem for several values of the viscosity parameter $\nu=\{0 ; 0.25 ; 0.5 ; 1 ; 2 ; \infty\}$.

the nonlinear advection-diffusion phenomena of fluid dynamics in one dimension [1], and also in combination with the effect of uncertainty $[13,19,25]$. The efficiency of uncertainty quantification in computational fluid dynamics applications is important, since deterministically fluid dynamics simulations can already result in high computational costs. The monomial chaos formulation for the Burgers equation is given in section 3.1. In section 3.2 numerical results for monomial chaos are presented.

3.1. Burgers' equation. In this section the one-dimensional steady nonlinear advection-diffusion problem known as the viscous Burgers equation is considered [1]. The Burgers equation for the velocity $u(x, \omega)$ in one dimension is given by

$$
u \frac{\partial u}{\partial x}-\nu \frac{\partial^{2} u}{\partial x^{2}}=0, \quad x \in[0,1],
$$

with an uncertain viscosity $\nu(\omega)$. The deterministic boundary conditions are $u(0, \omega)=$ 1 and $u(1, \omega)=0$. The solution of the deterministic variant of (3.1) is shown in Figure 1 for several values of the viscosity $\nu=\{0 ; 0.25 ; 0.5 ; 1 ; 2 ; \infty\}$. In Figure 1 also the sensor location $x_{\mathrm{sl}}=0.5$ is shown. The monomial chaos expansions for the uncertain viscosity $\nu(\omega)$ and the velocity $u(x, \omega)$ are

$$
\nu(\omega)=\sum_{j=0}^{1} \nu_{j} \Psi_{j}(\xi(\omega)), \quad u(x, \omega)=\sum_{i=0}^{\infty} u_{i}(x) \Psi_{i}(\xi(\omega))
$$

where $\xi(\omega)$ is a linear transformation of $\nu(\omega)$ to a standard domain and $\left\{\Psi_{i}(\xi)\right\}_{i=0}^{\infty}$ are monomials around $\xi(\omega)=\mu_{\xi}$ given by $(2.2)$. The expansion coefficients $\left\{\nu_{j}\right\}_{j=0}^{1}$ of the viscosity with a known uncertainty distribution are given by

$$
\nu_{j}=\left.\frac{1}{j !} \frac{\mathrm{d}^{j} \nu}{\mathrm{d} \xi^{j}}\right|_{\xi=\mu_{\xi}}, \quad j=0,1
$$

where $\left.\frac{\mathrm{d}^{j} \nu}{\mathrm{d} \xi^{j}}\right|_{\mu_{\xi}}$ is known. Substituting the monomial chaos expansions (3.2) into the Burgers equation (3.1) results in

$$
\sum_{i=0}^{\infty} \sum_{j=0}^{\infty} \Psi_{i}(\xi) \Psi_{j}(\xi) u_{j} \frac{\mathrm{d} u_{i}}{\mathrm{~d} x}-\sum_{i=0}^{\infty} \sum_{j=0}^{1} \Psi_{i}(\xi) \Psi_{j}(\xi) \nu_{j} \frac{\mathrm{d}^{2} u_{i}}{\mathrm{~d} x^{2}}=0 .
$$


Taking the $k$ th derivative of (3.4) with respect to $\xi$ for $\xi(\omega)=\mu_{\xi}$ and using the Leibniz identity and property (2.3) results in a differential equation for $u_{k}(x)$,

$$
\sum_{l=0}^{k}\left(\begin{array}{l}
k \\
l
\end{array}\right) u_{k-l}(x) \frac{\mathrm{d} u_{l}}{\mathrm{~d} x}-\sum_{l=\max \{0, k-1\}}^{k}\left(\begin{array}{l}
k \\
l
\end{array}\right) \nu_{k-l} \frac{\mathrm{d}^{2} u_{l}}{\mathrm{~d} x^{2}}=0, \quad k=0,1, \ldots
$$

Terms without $u_{k}(x)$ can be brought to the right-hand side of (3.5), which results in

$$
u_{0} \frac{\mathrm{d} u_{0}}{\mathrm{~d} x}-\nu_{0} \frac{\mathrm{d}^{2} u_{0}}{\mathrm{~d} x^{2}}=0, \quad k=0
$$

$$
u_{k} \frac{\mathrm{d} u_{0}}{\mathrm{~d} x}+u_{0} \frac{\mathrm{d} u_{k}}{\mathrm{~d} x}-\nu_{0} \frac{\mathrm{d}^{2} u_{k}}{\mathrm{~d} x^{2}}=-\sum_{l=1}^{k-1}\left(\begin{array}{l}
k \\
l
\end{array}\right) u_{k-l}(x) \frac{\mathrm{d} u_{l}}{\mathrm{~d} x}+k \nu_{1} \frac{\mathrm{d}^{2} u_{k-1}}{\mathrm{~d} x^{2}}, \quad k=1,2, \ldots
$$

As mentioned before, the equation for $k=0,(3.6 \mathrm{a})$, coincides with the deterministic problem for the mean value of the uncertain viscosity $\nu_{0}$. Equations (3.6b) form a decoupled set of equations for the higher-order monomial chaos coefficients $u_{k}(x)$, with $k=1,2, \ldots$, as function of $\left\{u_{j}(x)\right\}_{j=0}^{k-1}$ which can be solved sequentially for increasing $k$. These equations are linear in $u_{k}(x)$. The computational work for solving each equation of (3.6b) is equivalent to one Newton iteration for solving (3.6a). Therefore, monomial chaos results in relatively low computational costs per additional polynomial chaos order compared to the deterministic solve.

A $p$ th-order approximation of the solution for $u(x, \omega)$ can be obtained by truncating the monomial expansion for $u(x, \omega)$ in $(3.2)$ at $p$. The error estimates $(2.13)$ and (2.14) can be used to determine a suitable polynomial chaos order $p$ of the approximation. Equations (2.7) and (2.9) can be used to determine the approximation of the mean and the variance of the velocity $u(x, \omega)$.

3.2. Results for Burgers' equation. In this section results of the monomial chaos for the Burgers equation are presented. In section 4, the results of the monomial chaos approach are compared to results of the perturbation method, the Galerkin polynomial chaos method, and a nonintrusive polynomial chaos method as reviewed in section 1. For this comparison two error measures are used for the error in the mean $\varepsilon_{\mu_{\mathrm{u}}}(x)$ and the variance $\varepsilon_{\sigma_{\mathrm{u}}^{2}}(x)$ at the sensor location $x_{\mathrm{sl}}=0.5$ :

$$
\varepsilon_{\mu_{\mathrm{u}}}=\left|\frac{\mu_{\mathrm{u}}\left(x_{\mathrm{sl}}\right)-\mu_{\mathrm{u}, \mathrm{ref}}\left(x_{\mathrm{sl}}\right)}{\mu_{\mathrm{u}, \mathrm{ref}}\left(x_{\mathrm{sl}}\right)}\right|, \quad \varepsilon_{\sigma_{\mathrm{u}}^{2}}=\left|\frac{\sigma_{\mathrm{u}}^{2}\left(x_{\mathrm{sl}}\right)-\sigma_{\mathrm{u}, \mathrm{ref}}^{2}\left(x_{\mathrm{sl}}\right)}{\sigma_{\mathrm{u}, \mathrm{ref}}^{2}\left(x_{\mathrm{sl}}\right)}\right| .
$$

The reference solution is a Monte Carlo simulation based on $10^{6}$ realizations of the uncertain parameter $\nu(\omega)$ evenly spaced in sample space $\omega \in[0,1]$. Approximations of the probability distribution function and the probability density function are also presented. A second-order finite volume method is used to discretize the spatial domain. The nonlinear problem is solved using Newton linearization with an appropriate convergence criterion $\varepsilon_{\mathrm{nl}}=10^{-9}$ for the $L_{\infty}$-norm of the residual, which results for this problem in four Newton iterations.

The mean value of the uncertain input is assumed to be $\mu_{\nu}=1$. Probability distributions with either a finite or a (semi-)infinite support of the uncertain viscosity $\nu(\omega)$ are considered. The uniform distribution is chosen for the distribution on the finite domain. This corresponds to the assumption of an interval uncertainty, which 


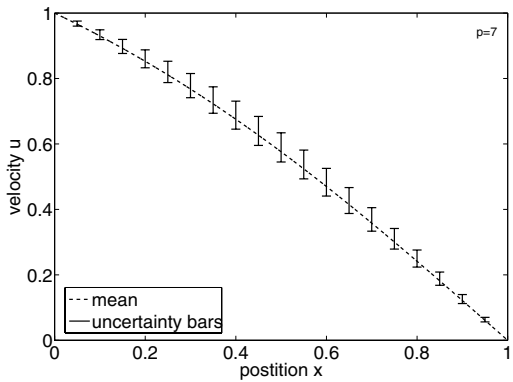

(a) mean and uncertainty bars

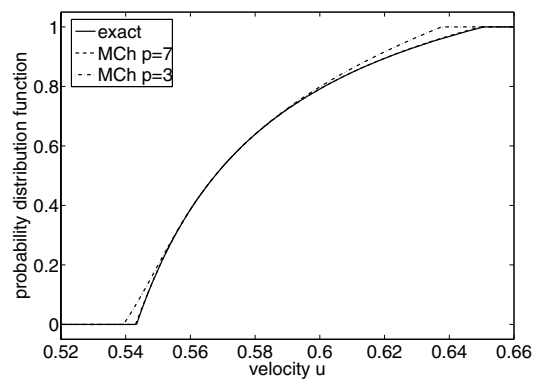

(b) distribution function at $x_{\mathrm{sl}}$

FIG. 2. Monomial chaos (MCh) results for the uniform input distribution.

is often used in practical applications in case not enough information is available to prescribe an uncertainty distribution. The input coefficient of variation for the uniform distribution is $\operatorname{cov}_{\nu}=0.3$. Physical uncertainties are often described using a normal distribution. Since the viscosity is a positive physical parameter, the lognormal distribution is selected instead of the normal distribution for the distribution on the (semi-)infinite domain. For the lognormal distribution an input coefficient of variation of $\operatorname{cov}_{\nu}=0.2$ is considered to limit the main parameter variations to the same range as for the uniform distribution. It has been verified that variation of the input coefficient of variation $\operatorname{cov}_{\nu}$ and the choice of the sensor location $x_{\mathrm{sl}}$ do not affect the results significantly in comparison with the other methods.

3.2.1. Results for the uniform input distribution. In Figure 2 the monomial chaos results for the uniform input distribution are presented. In Figure 2(a) the mean $\mu_{\mathrm{u}}$ and the $90 \%$ uncertainty intervals are given as function of $x$. The uncertainty is largest in the interior of the domain due to the deterministic boundary conditions. The uncertainty bars are asymmetrical with respect to the mean, which was expected from the deterministic parameter study of Figure 1. In Figure 2(b) the approximation of the probability distribution function at the sensor location $x_{\mathrm{sl}}$ is shown. The monomial chaos approximations for $p=3$ and $p=7$ are compared to the reference solution. The 7th-order approximation is very accurate, and the 3rd-order approximation results in a less accurate resolution of the tails of the distribution.

In Figure 3 the error convergence of the monomial chaos is given as a function of both the polynomial chaos order and the computational work for the uniform input distribution. In the same figure results for the perturbation method are given, which are discussed in section 4 . The mean and the variance converge on average exponentially as functions of polynomial chaos order; see Figure 3(a). The odd coefficients do not contribute to the approximation of the mean (2.7), since the central moments $\mu_{\nu, i}$ of $\nu(\omega)$ are zero for odd $i$. This is the reason for the staircase convergence of the approximation of the mean.

In Figure 3(b), the error convergence as a function of the computational work is given in terms of the equivalent number of deterministic solves. The error convergence with respect to computational work is four times faster than the convergence with respect to polynomial chaos order. For $p=0$ the monomial chaos results in a 


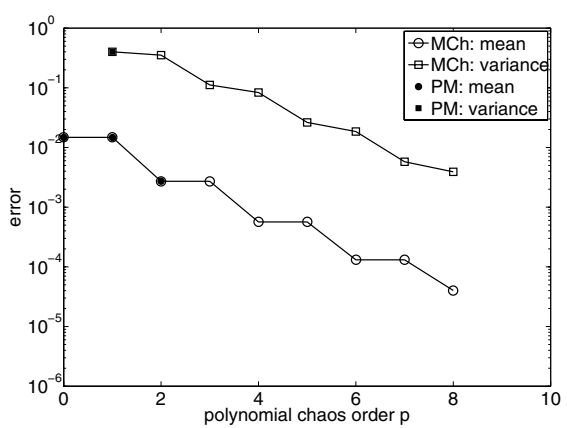

(a) polynomial chaos order

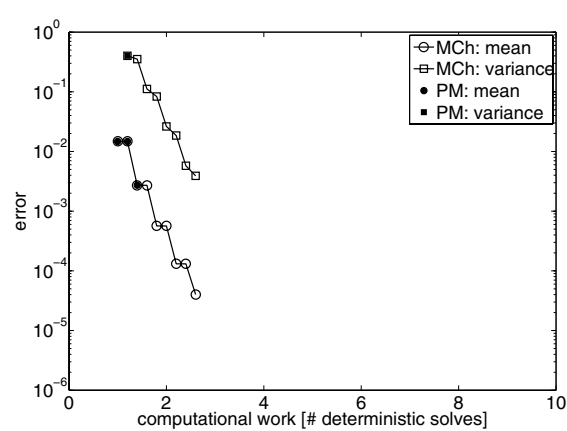

(b) computational work

FIG. 3. Error convergence of the monomial chaos (MCh) and the perturbation method (PM) for the uniform input distribution.

deterministic solve for the mean value of the uncertain input $\mu_{\nu}$. In this case four Newton iterations are required to solve the nonlinear problem. Per additional polynomial chaos order a linear problem has to be solved. The computational work for these linear solves is equivalent to one Newton iteration for the nonlinear problem. For an 8th-order monomial chaos approximation of the mean with an error of $1 \cdot 10^{-5}$ this results in computational costs equivalent to three deterministic solves. These results depend on the number of Newton iterations required for the deterministic problem.

3.2.2. Results for the lognormal input distribution. The results of the monomial chaos for the lognormal input distribution are given in Figure 4. In Figure 4(a) the monomial chaos approximation of the probability density function for $p=3$ and $p=7$ is compared to the reference solution at the sensor location $x_{\mathrm{sl}}$. Especially near the tails of the distribution the 7th-order approximation is more accurate than the 3rd-order approximation. In Figure 4(b) the weighted error in the approximation of the probability density function is shown. The error weighted with its probability is small near the point of highest probability, which corresponds approximately to $\mu_{\nu}$, and it vanishes in the tails.

In Figure 5 the error convergence of the monomial chaos is given for the lognormal input distribution. The mean and the variance converge, but the error convergence is less regular than for the uniform input distribution. Initially the convergence is less smooth due to the alternating over- and underestimation in combination with the asymmetrical input distribution. The first-order coefficient $u_{1}\left(x_{\mathrm{sl}}\right)$ has no contribution to the approximation of the mean, since the first-order central moment $\mu_{\nu, 1}$ of $\nu(\omega)$ is by definition zero. The error convergence with respect to computational work is again four times faster than with respect to polynomial order; see Figure 5(b).

4. Comparison with other methods. In this section the results of the monomial chaos for the Burgers equation are compared to the results of the perturbation method [10], the polynomial chaos method [3], and a nonintrusive polynomial chaos method [9]. An error convergence study with respect to the Monte Carlo reference solution is performed as a function of polynomial chaos order and computational work. For the Galerkin polynomial chaos method an optimal polynomial basis is constructed based on the input uncertainty distribution. For a nonintrusive polyno- 


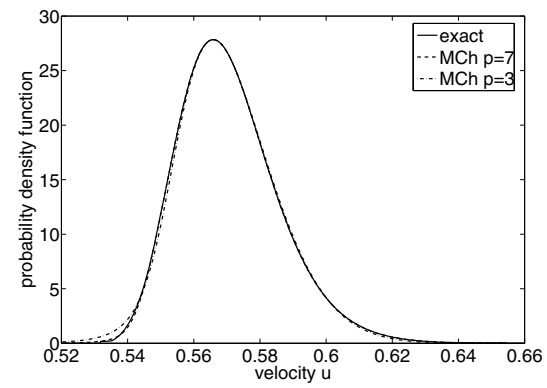

(a) probability density

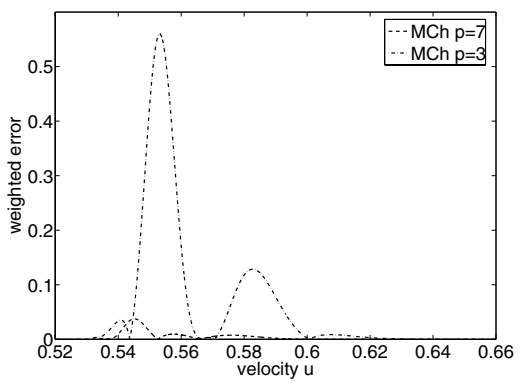

(b) weighted error

FIG. 4. Monomial chaos (MCh) results for the lognormal input distribution.

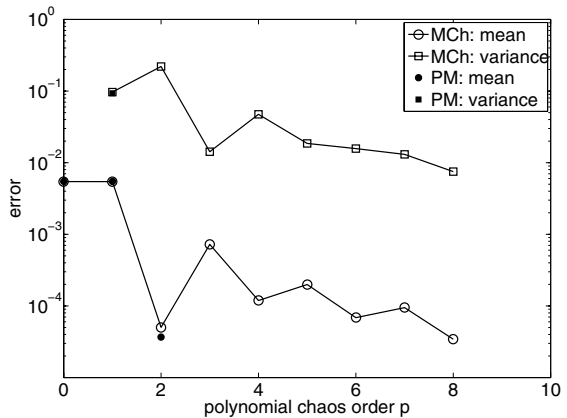

(a) polynomial order

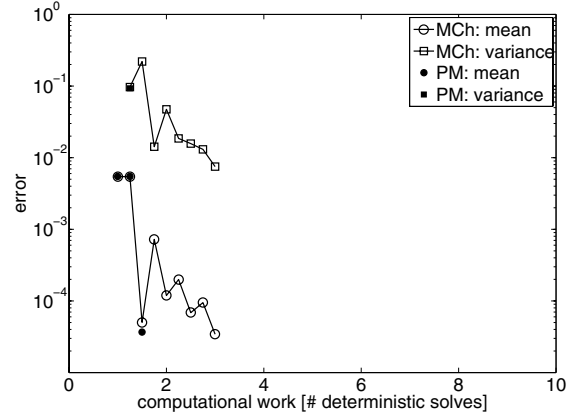

(b) computational work

FIG. 5. Error convergence of the monomial chaos (MCh) and the perturbation method (PM) for the lognormal input distribution.

mial chaos method the solution is not unique since the samples $\xi_{k}$ in random space in (1.9) can be chosen arbitrarily [9]. Here the sampling points are chosen uniformly distributed in $\omega$.

4.1. Comparison with the perturbation method. In contrast with the monomial chaos approach, the perturbation method results only in low-order approximations of the mean and the variance. These results are compared to the results of the monomial chaos in Figures 3 and 5 for the uniform and lognormal input distribution, respectively. The results of the perturbation method are similar to those of the monomial chaos for $p=0,1,2$. Higher-order monomial chaos approximations for the uniform input distribution are orders of magnitude more accurate than those of the perturbation method. This demonstrates that the monomial chaos method can be viewed as an extension of the perturbation method to higher-order approximations of the mean, the variance, and the distribution function. Also the computational costs of the monomial chaos approach and the perturbation method of the same order are 


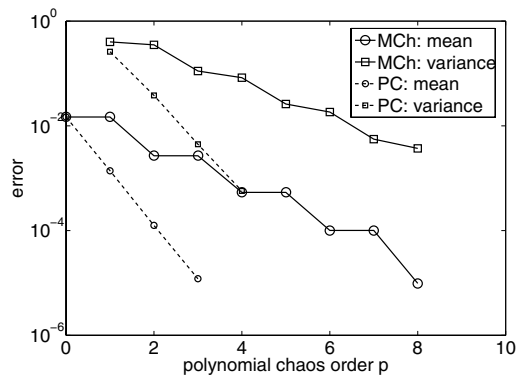

(a) polynomial order

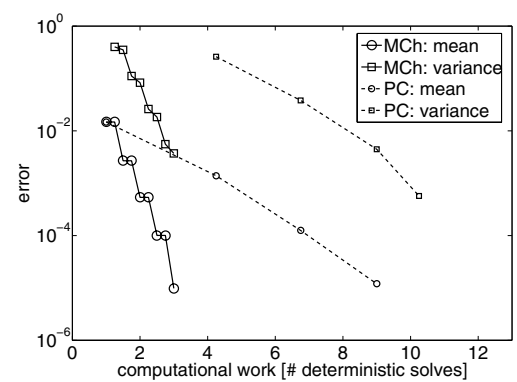

(b) computational work

FIG. 6. Error convergence of the monomial chaos (MCh) and the Galerkin polynomial chaos $(P C)$ for the uniform input distribution.

similar; see Figures 3(b) and 5(b). For higher-order approximations the monomial chaos approach maintains these low computational costs per additional polynomial chaos order.

4.2. Comparison with the Galerkin polynomial chaos method. The mean and variance approximations of the monomial chaos approach and the Galerkin polynomial chaos method are compared in Figures 6 and 7 for the uniform and lognormal input distribution, respectively. In terms of polynomial chaos order, the Galerkin polynomial chaos method results in exponential and faster convergence than the monomial chaos approach; see Figures 6(a) and 7(a). However, solving the coupled set of nonlinear equations in the Galerkin polynomial chaos method results in a relatively fast increase of computational work per polynomial chaos order in comparison with the monomial chaos approach. Let $p$ be the polynomial chaos order, $n_{\mathrm{N}}$ be the number of Newton iterations for solving the nonlinear problem, and $n_{\mathrm{GS}}$ be the number of Gauss-Seidel iterations for solving the coupled system of the Galerkin polynomial chaos. Then the monomial chaos approach results in an amount of computational work equivalent to $\left(\frac{p}{n_{\mathrm{N}}}+1\right)$ deterministic solves. The computational work for the Galerkin polynomial chaos is equivalent to $n_{\mathrm{GS}}(p+1)$ deterministic solves. Therefore, in this case the monomial chaos approach converges as a function of computational work by approximately a factor of three faster than the Galerkin polynomial chaos method; see Figures 6(b) and 7(b).

4.3. Comparison with a nonintrusive polynomial chaos method. In Figures 8 and 9 the error convergence of the monomial chaos and a nonintrusive polynomial chaos method is compared for the uniform and lognormal distribution, respectively. The nonintrusive polynomial chaos method achieves a slightly higher error convergence rate as a function of the polynomial chaos order; see Figures 8(a) and 9(a). The absolute errors are approximately of the same order of magnitude as those of the monomial chaos. The computational work of the nonintrusive polynomial chaos method per additional polynomial chaos order is equivalent to a nonlinear deterministic solve. So, the nonintrusive polynomial chaos results in an amount of computational work equivalent to $(p+1)$ deterministic solves compared to $\left(\frac{p}{n_{\mathrm{N}}}+1\right)$ for the monomial chaos approach. This results in this case in an approximately two times higher 


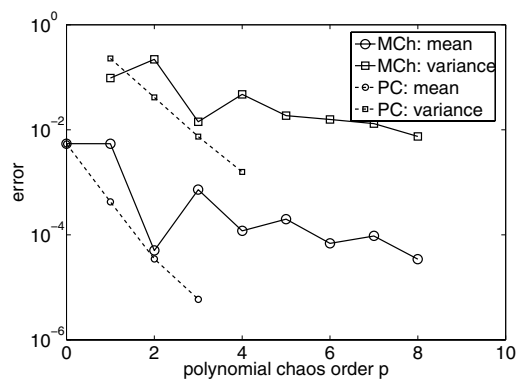

(a) polynomial order

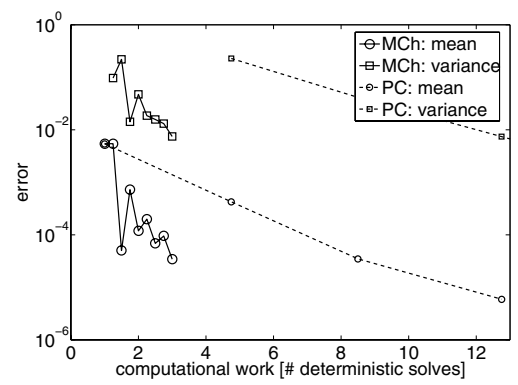

(b) computational work

FIG. 7. Error convergence of the monomial chaos (MCh) and the Galerkin polynomial chaos $(P C)$ for the lognormal input distribution.

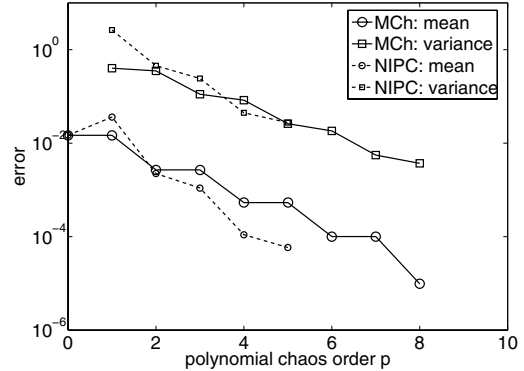

(a) polynomial order

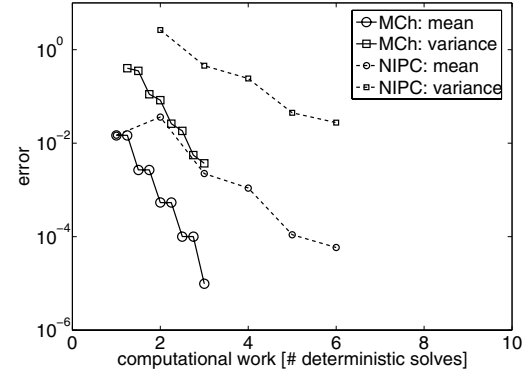

(b) computational work

FIG. 8. Error convergence of the monomial chaos (MCh) and a nonintrusive polynomial chaos method (NIPC) for the uniform input distribution.

error convergence rate as a function of computational work for the monomial chaos approach compared to the nonintrusive polynomial chaos method; see Figures 8(b) and $9(\mathrm{~b})$.

5. Application to two-dimensional boundary layer flow. In this section the monomial chaos approach is applied to a two-dimensional incompressible boundary layer flow as a standard test problem of computational fluid dynamics [14]. Uncertainty quantification in computational fluid dynamics can be highly expensive in practical applications due to the large computational work already involved in solving the deterministic problem. Monomial chaos can be a computationally efficient alternative for uncertainty quantification in this type of problem.

For two-dimensional flow along a flat plate the Navier-Stokes equations of viscous fluid dynamics reduce to the nonlinear two-dimensional incompressible boundary layer 


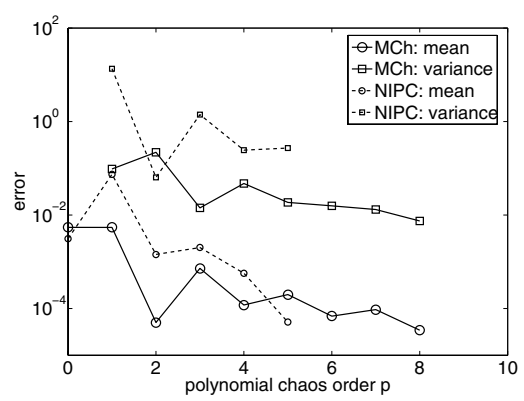

(a) polynomial order

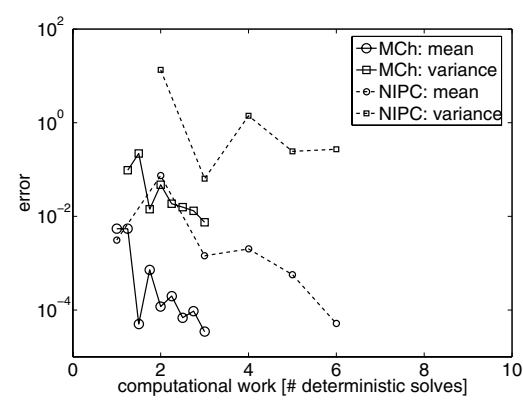

(b) computational work

FIG. 9. Error convergence of the monomial chaos (MCh) and a nonintrusive polynomial chaos method (NIPC) for the lognormal input distribution.

equations

$$
\begin{gathered}
\frac{\partial u}{\partial x}+\frac{\partial v}{\partial y}=0 \\
\rho u \frac{\partial u}{\partial x}+\rho v \frac{\partial u}{\partial y}-\mu \frac{\partial^{2} u}{\partial x^{2}}=0
\end{gathered}
$$

where $u$ and $v$ are the velocity components parallel and perpendicular to the plate, respectively. The flow is assumed to be laminar, the pressure gradient normal to the plate is neglected, and the density $\rho$ and viscosity $\mu$ are assumed to be uniform and independent of temperature. The boundary layer equations describe the conservation of mass (5.1a) and the conservation of momentum in the free stream direction (5.1b). The flat plate is aligned with the free stream direction $x$; see Figure 10. The free stream velocity $u_{\infty}$ equals unity, and the density at standard sea level conditions, $\rho_{\mathrm{ISA}}=1.225 \mathrm{~kg} / \mathrm{m}^{3}$, is used. The computational domain has length $1 \mathrm{~m}$ and height $0.05 \mathrm{~m}$ and is discretized with cells of length $\Delta x=1 \cdot 10^{-3} \mathrm{~m}$ with an aspect ratio of 2 . A mixed upwind-central discretization is used. The flat plate of length $0.9 \mathrm{~m}$ starts at $x=0.1 \mathrm{~m}$. To solve the deterministic problem, eight Newton iterations were required to reach the convergence criterion of $\varepsilon_{\mathrm{u}}=1 \cdot 10^{-4}$ in the $\mathrm{L}_{1}$-norm.

The uncertainty is introduced in terms of an uncertain dynamic viscosity coefficient $\mu(\omega)$. The uncertainty is described by a lognormal distribution, since viscosity is a positive physical parameter. The mean value is the viscosity at standard sea level conditions, $\mu_{\mathrm{ISA}}=1.789 \cdot 10^{-5} \mathrm{~kg} / \mathrm{ms}$, and the coefficient of variation is $\operatorname{cov}_{\mu}=5 \%$. The effect of the uncertainty in the viscosity on the velocity field and the drag of the flat plate are considered.

A third-order monomial chaos expansion is employed to solve for the uncertainty propagation in the boundary layer flow. The uncertain velocity components $u(x, y, \omega)$ and $v(x, y, \omega)$ and the viscosity $\mu(\omega)$ are expanded in a monomial chaos expansion. After substitution and differentiation of the governing equations (5.1), the uncertainty 


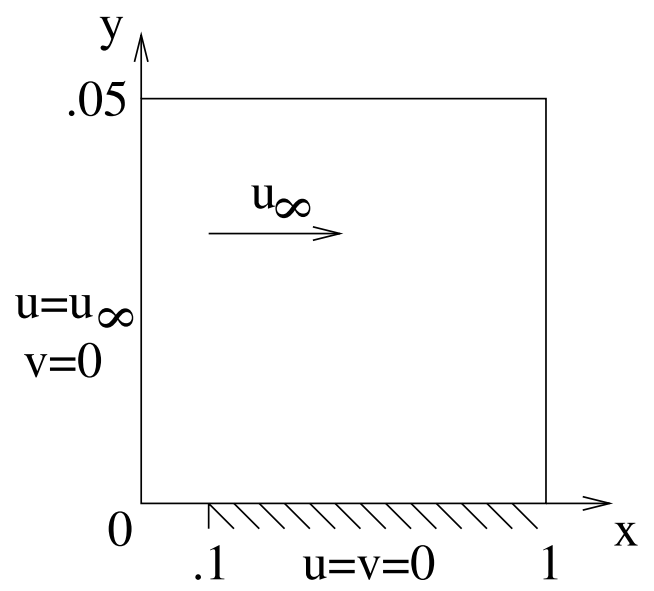

FIG. 10. The two-dimensional boundary layer flow problem.

quantification problem is given by

$$
\begin{gathered}
\frac{\partial u_{k}}{\partial x}+\frac{\partial v_{k}}{\partial y}=0 \\
\sum_{l=0}^{k}\left(\begin{array}{c}
k \\
l
\end{array}\right) u_{k-l} \frac{\partial u_{l}}{\partial x}+\sum_{l=0}^{k}\left(\begin{array}{c}
k \\
l
\end{array}\right) v_{k-l} \frac{\partial u_{l}}{\partial y}-\sum_{l=\max \{0, k-1\}}^{k}\left(\begin{array}{c}
k \\
l
\end{array}\right) \mu_{k-l} \frac{\partial^{2} u_{l}}{\partial x^{2}}=0
\end{gathered}
$$

for $k=\{0,1,2,3\}$. In Figure 11 the results for the mean $\mu_{\mathrm{u}}(x, y)$ and the standard deviation $\sigma_{\mathrm{u}}(x, y)$ of the $u$-velocity field are shown. The presence of the flat plate results in a typical boundary layer behavior of the mean $u$-velocity field; see Figure 11(a). The standard deviation of the $u$-velocity field has local maxima inside the boundary layer and near the leading edge of the flat plate. It vanishes both near the flat plate further downstream and in the outer flow; see Figure 11(b). The error estimates (2.13) and (2.14) estimate a maximum error of $4 \cdot 10^{-6}$ and $8 \cdot 10^{-5}$ in the mean and variance field, respectively.

The drag $F_{\text {drag }}(\omega)$ of the two-sided flat plate is a function of the uncertain viscosity $\mu(\omega)$ and the uncertain velocity gradient at the wall $\left.\frac{\partial u}{\partial y}\right|_{y=0}(\omega)$,

$$
F_{\operatorname{drag}}(\omega)=2 \int_{L} \tau_{\mathrm{w}}(\omega) \mathrm{d} x=\left.2 \int_{0.1}^{1} \mu(\omega) \frac{\partial u}{\partial y}\right|_{y=0}(\omega) \mathrm{d} x,
$$

where $\tau_{\mathrm{w}}(\omega)$ is the skin friction. In Figure 12 the third-order monomial chaos approximation of the uncertainty distribution of the drag is shown. In Figure 12(a) the probability distribution function is compared to a Monte Carlo simulation based on 100 realizations uniformly sampled in $\omega$. The results show good agreement. In Figure 12(b) the error in the distribution function weighted by its probability is given. The error is minimal for the drag corresponding to the mean value of the viscosity and vanishes in the tails.

The additional computational costs of the presented uncertainty quantification are equivalent to less than a deterministic solve. As mentioned before, solving the nonlinear deterministic problem requires eight Newton iterations. The third-order monomial chaos results in three linear solves in addition to the deterministic solve 


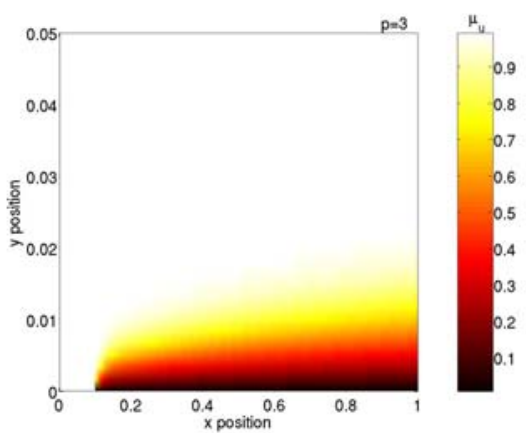

(a) mean $\mu_{u}(x, y)$

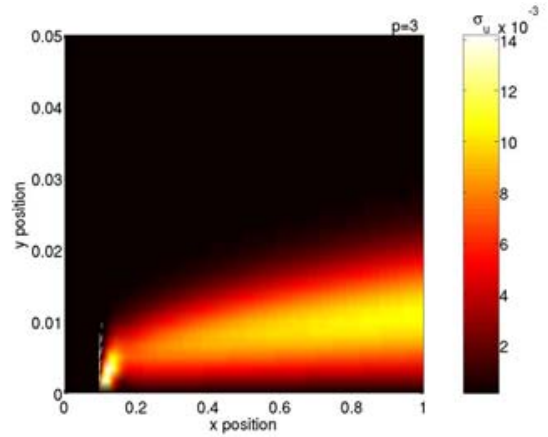

(b) standard deviation $\sigma_{\mu}(x, y)$

FIG. 11. Uncertain u-velocity field in the two-dimensional boundary layer flow problem subject to uncertain viscosity.

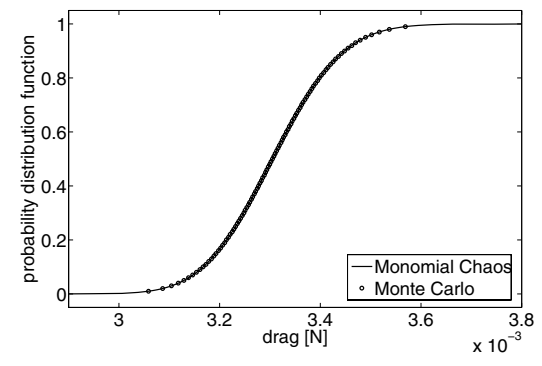

(a) probability distribution function

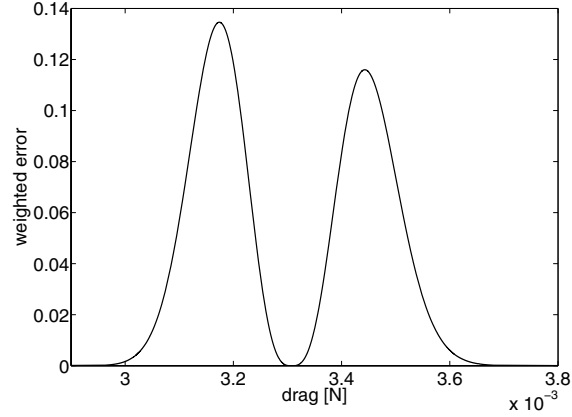

(b) weighted error

FIG. 12. Uncertainty distribution of the drag in the two-dimensional boundary layer flow problem subject to uncertain viscosity.

for the mean value of the uncertain input parameter. So, the additional computational costs for the uncertainty quantification using monomial chaos are in this case equivalent to $\frac{3}{8}$ of the computational cost for solving the deterministic problem. Performing uncertainty quantification in computationally intensive practical applications is economically feasible with this order of computational costs.

6. Summary. A monomial chaos approach is proposed for efficient uncertainty quantification in computationally intensive nonlinear problems. The proposed approach employs a polynomial chaos expansion with monomials as basis functions. The equations for the deterministic coefficients are obtained by differentiating the governing equations. Propagating uncertainty through nonlinear equations can be computationally intensive for other polynomial chaos methods. It usually results in a set of nonlinear equations which can be coupled. The proposed monomial chaos approach results in a decoupled set of linear equations even for problems involving 
polynomial nonlinearities. This reduces the computational work per additional polynomial chaos order to the equivalence of a single Newton iteration. Error estimates for the monomial chaos approach have been presented. It has been demonstrated numerically that the monomial chaos approach can achieve a 2-3 times faster convergence as a function of computational work than other polynomial chaos methods. Application to a two-dimensional flow problem demonstrated that the additional computational work for performing an uncertainty quantification using monomial chaos can be smaller than a single deterministic solve.

\section{REFERENCES}

[1] D. A. Anderson, J. C. Tannehill, And R. H. Pletcher, Computational Fluid Mechanics and Heat Transfer, Ser. Comput. Methods Mech. Thermal Sci., McGraw-Hill, New York, 1997.

[2] R. H. Cameron And W. T. Martin, The orthogonal development of nonlinear functionals in series of Fourier-Hermite functionals, Ann. Math., 48 (1947), pp. 385-392.

[3] R. G. Ghanem and P. Spanos, Stochastic Finite Elements: A Spectral Approach, SpringerVerlag, New York, 1991.

[4] R. G. Ghanem And S. F. Wojtkiewicz, eds., Special issue on uncertainty quantification, SIAM J. Sci. Comput., 26 (2004), issue 2.

[5] A. Greenbaum, Iterative Methods for Solving Linear Systems, SIAM, Philadelphia, 1997.

[6] J. M. Hammersley and D. C. Handscomb, Monte Carlo Methods, Methuen's Monographs on Applied Probability and Statistics, Fletcher \& Son, Norwich, CT, 1964.

[7] E. J. Haug, K. Choi, and V. Komkov, Design Sensitivity Analysis of Structural Systems, Academic Press, Orlando, FL, 1986.

[8] C. Hirsch, Numerical Computation of Internal and External Flows, Vol. 1: Fundamentals of Numerical Discretization, Wiley, Chichester, UK, 1988.

[9] S. Hosder, R. W. Walters, and R. Perez, A non-intrusive polynomial chaos method for uncertainty propagation in CFD simulations, in Proceedings of the 44th AIAA Aerospace Sciences Meeting and Exhibit, Reno, NV, 2006, AIAA-2006-891.

[10] M. Kleiber And T. D. Hien, The Stochastic Finite Element Method, John Wiley and Sons, New York, 1992.

[11] M. LoÈve, Probability Theory, 4th ed., Springer-Verlag, New York, 1977.

[12] J.-N. Mahieu, S. Étienne, D. Pelletier, and J. Borggaard, A second-order sensitivity equation method for laminar flow, Int. J. Comput. Fluid D, 19 (2005), pp. 143-157.

[13] L. Mathelin And O. P. Le MaîtRe, A posteriori error analysis for stochastic finite element solutions of fluid flows with parametric uncertainties, in Proceedings of the European Conference on Computational Fluid Dynamics (ECCOMAS CFD 2006), Egmond aan Zee, The Netherlands, P. Wesseling, E. Oñate, and J. Périaux, eds., TU Delft, The Netherlands, 2006.

[14] H. T. Schlichting and K. Gersten, Boundary-Layer Theory, Springer, Berlin, 2000.

[15] G. I. SCHUËLlER, ED., A state-of-the-art report on computational stochastic mechanics, Prob. Engrg. Mech., 12 (1997), pp. 197-321.

[16] L. G. Stanley And D. L. Stewart, Design Sensitivity Analysis: Computational Issues of Sensitivity Equation Methods, Frontiers Appl. Math. 25, SIAM, Philadelphia, 2002.

[17] É Turgeon, D. Pelletier, And J. BorggaArd, A general continuous sensitivity equation formulation for complex flows, Numer. Heat Transfer B, 42 (2002), pp. 485-408.

[18] R. W. Walters, Towards stochastic fluid mechanics via polynomial chaos-Invited, in Proceedings of the 41st AIAA Aerospace Sciences Meeting and Exhibit, Reno, NV, 2003, AIAA-2003-0413.

[19] R. W. Walters and L. Huyse, Uncertainty Analysis for Fluid Mechanics with Applications, NASA research report NASA/CR-2002-21149, NASA Langley Research Center, Hampton, VA, 2002; available online from http://historical.ncstrl.org/tr/pdf/icase/TR-2002-1.pdf.

[20] X. Wan And G. E. Karniadakis, Beyond Wiener-Askey expansions: Handling arbitrary PDFs, J. Sci. Comput., 27 (2006), pp. 455-464.

[21] N. Wiener, The homogeneous chaos, Amer. J. Math., 60 (1938), pp. 897-936.

$[22]$ J. A. S. Witteveen And H. BisL, Modeling arbitrary uncertainties using Gram-Schmidt polynomial chaos, in Proceedings of the 44th AIAA Aerospace Sciences Meeting and Exhibit, Reno, NV, 2006, AIAA-2006-896. 
[23] D. Xiu and G. E. Karniadakis, The Wiener-Askey polynomial chaos for stochastic differential equations, SIAM J. Sci. Comput., 24 (2002), pp. 619-644.

[24] D. XIU AND G. E. KARNiAdakis, Modeling uncertainty in flow simulations via generalized polynomial chaos, J. Comput. Phys., 187 (2003), pp. 137-167.

[25] D. XiU And G. E. Karniadakis, Uncertainty modeling of Burgers' equation by generalized polynomial chaos, in Computational Stochastic Mechanics, 4th International Conference on Computational Stochastic Mechanics, Corfu, Greece, 2002, P. D. Spanos and G. Deodatis, eds., Millpress, Rotterdam, The Netherlands, 2003, pp. 655-661.

Copyright $@$ by SIAM. Unauthorized reproduction of this article is prohibited. 